Archives de sciences sociales des religions

180 | octobre-décembre 2017

Bulletin bibliographique

\title{
De la sociologie-historique du protestantisme à la sociologie historique de la laïcité
}

Jean Baubérot

\section{OpenEdition}

1 Journals

Édition électronique

URL : https://journals.openedition.org/assr/29719

DOI : 10.4000/assr.29719

ISSN : $1777-5825$

Éditeur

Éditions de l'EHESS

Édition imprimée

Date de publication : 1 décembre 2017

Pagination : 51-68

ISSN : 0335-5985

\section{Référence électronique}

Jean Baubérot, « De la sociologie-historique du protestantisme à la sociologie historique de la laïcité », Archives de sciences sociales des religions [En ligne], 180 | octobre-décembre 2017, mis en ligne le 01 décembre 2019, consulté le 22 septembre 2021. URL : http://journals.openedition.org/assr/29719 ; DOI : https://doi.org/10.4000/assr.29719 


\section{Jean Baubérot}

\section{De la sociologie historique du protestantisme à celle de la laïcité}

Ce titre appelle à retracer un itinéraire intellectuel. En effet, ma carrière à l'École Pratique des hautes études, commencée en 1967, m'a conduit à devenir titulaire de la chaire «Histoire et sociologie des protestantismes» (1978-1991), et ensuite d'une nouvelle chaire, "Histoire et sociologie de la laïcité » (1991-2007), la première spécialisée dans cet objet d'études. Ce parcours s'est effectué en constante interaction avec le milieu de la recherche, française et internationale. Il faut citer, outre l'EPHE elle-même, le Groupe de Sociologie des Religions, sa revue les Archives (Baubérot, 2006a), et le séminaire de Jean-Marie Mayeur, qualifié ensuite de «moment Créteil» de l'histoire religieuse contemporaine ${ }^{1}$. La progressive émergence d'une sociologie historique de la laïcité est une histoire collective, dont la création de la chaire Histoire et sociologie de la laïcité, et trois ans plus tôt, la fondation d'une Jeune Équipe éponyme à l'EPHE, ont été les premiers aboutissements institutionnels.

\section{Le GSR, et l'idée d'une sociologie possible de la laïcité}

Créé en 1954, le Groupe de Sociologie des Religions devient une formation propre du CNRS en 1971. Je le fréquente ${ }^{2}$. Un processus international de transformation de la sociologie religieuse (plus ou moins liée à des stratégies pastorales) en sociologie des religions s'est opéré dans les années 1950 et 1960. Dans le cadre de cette déconfessionnalisation et de l'inscription dans la recherche universitaire, des théories de la sécularisation ont été élaborées. Celle-ci est définie comme l' " autonomisation progressive de secteurs sociaux qui échappent à la domination des significations et des institutions religieuses» (Berger, Luckmann, 1966: $72 \mathrm{sq}$ ). Avec ce concept-clé, les sociologues de la religion réussissent une prouesse: plus leur objet semble décliner empiriquement, plus il prend une importance théorique. Si la religion paraît marginale dans la «société moderne", la sécularisation en constitue la caractéristique essentielle.

1. Voir Cuchet, Pelletier, 2016. Outre C. Langlois et moi-même, participèrent à ce séminaire A. Encrevé, E. Fouilloux, J. Prévotat, etc.

2. J'en deviendrai officiellement membre en 1974. 


\section{«Une société se laïcise et se sécularise... »}

Pourtant, tel le village d'Astérix, le GSR fait de la résistance. Un exemple significatif : l'étude de Peter Berger et Thomas Luckmann, "Secularization and pluralism ", de 1966 est publiée en français par les Archives sous le titre d' "Aspects sociologiques du pluralisme » (Berger, Luckmann, 1967). Le terme de "sécularisation » a disparu alors que, sur le plan international, la «sécularisation devient un paradigme qui englobe conceptuellement le pluralisme (Tschannen, 1991; Baubérot, Milot, 2011): "De gré ou de force, les chercheurs ont dû examiner les questions se rapportant au processus de sécularisation»(Cipriani, 1981: 144).

Peut-être le fait que la constitution du Groupe ait précédé l'essor des théories de la sécularisation constitue une des raisons de leur faible influence. Il n'en sera pas de même dans la génération qui suivra celle des pères-fondateurs ${ }^{3}$. Toujours est-il que le constat en est frappant dans le bilan de "Quinze ans de vie et de travail» (GSR = Poulat, 1969) écrit en 1969: peu d'études concernent la sécularisation. De plus, Émile Poulat, alors directeur du Groupe, propose une définition limitée de cette notion, grâce à la distinction faite entre "se séculariser » et «se laïciser ", dans le cadre d'une typologie où une société:

1. «Se laïcise quand elle se soustrait au contrôle ou à la sujétition d'un clergé (phénomène ancien, périodique, et non spécifiquement catholique);

2. se sécularise quand elle se détermine sur un type de rationalité dégagé de toute conception religieuse [...] sans d'ailleurs nécessairement rejeter la religion et encore moins l'idéologie;

3. se modernise quand elle s'adapte aux modes de vie et aux formes de pensée qu'entraine cette rationalité;

4. se déchristianise quand elle se détache de la mouvance chrétienne».

«Le jeu de ces phénomènes, conclut Poulat, doit être analysé aux deux niveaux de la société globale et de l'institution religieuse, [...] à la fois en terme de perte et de gain et en terme de changement [...] qui implique des contraintes, des stratégies, des révisions.» (ibid.: 39 sq.)

Cette typologie pose quelques problèmes théoriques. Pourtant, il s'agit d'une piste intéressante car elle indique que l'on pourrait distinguer des processus idéal-typiques différents, dont il serait possible d'analyser les écarts, les similitudes, les interférences... sauf qu'il s'agit, en fait, d'un simple mode de classement de travaux des membres du GSR. D'autre part, la perspective de la «laïcisation » (le terme est utilisé) ne se retrouve que dans deux études, rédigées par Poulat lui-même (Poulat, 1958, 1960) ${ }^{4}$.

3. Voir, par exemple, les travaux de D. Hervieu-Léger et de J.-P. Willaime.

4. Poulat signale également l'ouvrage d'un universitaire "proche du Groupe», J.-M. Mayeur. Cf. infra. 


\section{«La laïcité comme fait social»}

Une piste est esquissée, sans être approfondie. Dix ans plus tard, on retrouve un souci équivalent dans le compte-rendu de François-André Isambert sur la Conférence de la Société Internationale de Sociologie des Religions (SISR) à Venise (1979), consacrée à «Religion et politique» (Isambert, 1980)5 . Il reproche aux communicants "de relier l'économique et le culturel sans s'apercevoir d'un certain vide laissé au niveau du plan médian de l'exercice du pouvoir ». Seuls, selon lui, deux exposés se sont référés à « la laïcité comme caractère des États [...] modernes ». Or, pour Isambert, la laïcité n'est pas une réalité "contingente » mais "une véritable fonction, plus juridique ici, plus culturelle là ", diversifiée selon les pays. Il regrette donc que «la laïcité comme fait social» n'ait guère eu droit de cité. Mais, ajoute-t-il, «le danger aurait pu être que la laïcité à la française soit prise comme prototype de toute laïcité».

Ce propos pointe un impensé du paradigme de la sécularisation: la sousestimation de la structuration des sociétés en États, lieux de la régulation politique du religieux, ce qui était logique dans le cadre de la croyance en un déclin et en une privatisation croissante de la religion. Le programme esquissé par Isambert reste, cependant, au niveau des intentions. Sur un plan international, le paradigme s'est imposé comme un facteur de notoriété professionnelle. Ainsi, longtemps réticent, David Martin, devenu président de la SISR, publie une "théorie générale de la sécularisation » (D. Martin, 1978). Une prise en compte du politique est présente, ce qui rend son étude originale. Mais elle se traduit par des expressions peu formalisées (disestablishment, the disconnection of Church and State, the Church unhinged from the State, distinguishing Church from State). Martin fournit des matériaux pour une problématique de la laïcité (ou du secularism), sans l'établir véritablement. On a l'impression que les contraintes du paradigme commencent à supplanter son intérêt heuristique.

\section{De Dobbelaere à Poulat}

À l'époque, la tentative de conceptualisation du terme de "laïcisation », dans le champ de la sociologie ${ }^{6}$, n'est pas venue du GSR mais de Karel Dobbelaere (Dobbelaere, 1981). Le chercheur belge considère la sécularisation comme un «concept multidimensionnel » qu'il décompose en trois niveaux: la transformation interne de la religion (religious change), la baisse de participation (involvement) religieuse et, le plus important des trois, la laïcisation (laicization), processus de différenciation structurelle et fonctionnelle des institutions où la religion devient une institution parmi d'autres. Cela suscite la réserve de sociologues anglophones

5. Isambert a quitté le GSR mais continue d'être en relation avec lui, grâce aux Archives.

6. En effet, des juristes américains ont formalisé, dès les années 1960, le secular state, par trois formes, en interaction, de relations sociojuridiques (la liberté de religion et par rapport à la religion; la citoyenneté sans condition de religion; la séparation de la religion et de l'État). À cet égard, voir Smith, 1963; Galanter, 1965. Mais ces études ne semblent pas avoir été lues par les sociologues. Personnellement, je les ai découvertes seulement en 1998. 
et peu d'écho au GSR ${ }^{7}$. Dobbelaere renonce à développer davantage, avant d'effectuer une nouvelle tentative en 2009 (Dobbelaere, 2009).

En 1986, Danièle Hervieu-Léger et Françoise Champion présentent une synthèse des études de sociologie de la religion effectuées en France. Elle reste centrée sur un paradigme sécularisation-modernité. La notion de "laïcisation » apparaît marginalement ${ }^{8}$. On va en reparler. Mais le contexte est en train de changer, rappelant aux sociologues que si les diagnostics peuvent avoir une certaine scientificité, les pronostics sont plus hasardeux. Les rapports entre politique et religion (re)deviennent à l'ordre du jour avec l'émergence de la nouvelle droite chrétienne aux États-Unis, les conséquences de la révolution iranienne, l'irruption de l' «islam politique ", le rôle joué par des acteurs «religieux» dans la chute de régimes communistes. En 1994, l'ouvrage de José Casanova, Publics Religions in the Modern World, amorce un tournant théorique, à un niveau international, critique à l'égard du paradigme de la sécularisation.

Dès $1988^{9}$, cependant, Émile Poulat infléchit son parcours d'historien-sociologue du catholicisme pour "comprendre le passage d'une société fondée sur le principe de catholicité à une société qui serait désormais appuyée sur le principe de modernité » (Poulat, 1994). Il trace une piste précieuse, dans son étude Liberté-laïcité ${ }^{10}$. En lien avec son œuvre antérieure (Poulat, 1977; Gugelot, 2016), il étudie les référentiels de "l'intransigeantisme» catholique et de "l'explosion libérale» lors de «la guerre des deux France». Il insiste sur l'enjeu conflictuel majeur des conceptions divergentes de la «liberté »; liberté en tant que vécu idéal, instrument de combat et/ou de pouvoir. Il oppose la conception catholique de «la liberté des consciences ", où "la conscience [de chacun] se soumet librement à l'autorité reconnue de la religion» et la «liberté de conscience» où la conscience individuelle devient "l'instance suprême, qui se détermine souverainement en matière de croyance et de religion " ${ }^{11}$. Comme l'indique Philippe Portier, dans son étude consacrée aux travaux de Poulat sur la laïcité ${ }^{12}$, le passage du principe de catholicité à celui de modernité "renvoie à deux moments différents de la relation Église/État [...] : dans la première, "la République est dans l'Église", dans la seconde, "l’Église est dans la République” (Portier, 2016a: 91).

7. Pour ma part, je suis en train d'élaborer la notion de «seuils de laïcisation " qui autonomise complètement la laïcisation de la sécularisation. Cf. infra.

8. Hervieu-Léger, 1986. En fait, il s'agit surtout d'études sur le christianisme français.

9. J'ai été «l'élève» de Poulat de 1972 à 1978.

10. Poulat, 1988. Poulat n'a pas systématiquement poursuivi ces pistes théoriques dans ses travaux ultérieurs.

11. Pour Poulat, la «liberté de conscience» est liée à la modernité libérale issue des Lumières. C'est une acceptation implicite des théories de la sécularisation. Pour M. Weber, l'affaire est plus complexe et, à côté de «la raison d'État purement politique» et du «mercantilisme » qui ont favorisé l' "idée de tolérance », il mentionne la réclamation de la «liberté de conscience [...] pour des raisons religieuses positives » par «la version radicale de la piété calviniste » et la Confession des baptistes de 1644 (M. Weber, 2003: 155).

12. Poulat publie ensuite d'autres études: Poulat, 2003, 2010. 


\section{L'étude de la loi de 1905 par Jean-Marie Mayeur et le «moment Créteil ».}

C'est la Révolution française qui a mis en route ce changement, mais c'est la loi de 1905, séparant les Églises et l'État, qui le symbolise. Or, longtemps, c'est l'étude de la laïcité scolaire qui a été privilégiée par les historiens et intégrée dans une histoire de l'école. Encore en 2005, Jean-Marie Mayeur peut écrire, par euphémisme: "L'histoire de la séparation n'a pas suscité une bibliographie considérable» (Mayeur, 2005: 9). De fait, malgré l'ouvrage d'Antonin Debidour publié peu après le vote de la loi ${ }^{13}$, très vite la mémoire collective prend le pas sur l'approche historienne. Émile Combes remplace Aristide Briand comme l'auteur de la loi de séparation. Évoquer la «séparation du petit père Combes » renvoie soit à une loi «persécutrice » à l'égard de l'Église catholique, soit à l'heureuse victoire, sans compromis, d'une laïcité anticléricale. Par transmission orale, et à travers une maigre historiographie polémique, légende noire et légende dorée ont colporté la même narration, avec ces jugements antagoniques. Longtemps, ces deux traditions se sont confortées, donnant valeur d'évidence à leur récit commun ${ }^{14}$. En 1964, l'imaginaire de la légende noire imprègne encore des historiens catholiques tels André Latreille et René Rémond (1964: 504-513) ${ }^{15}$, alors même que le Concile Vatican II met en œuvre un aggiornamento de l'Église catholique.

\section{L'approche de la Séparation dans une optique d'histoire du catholicisme}

Deux ans plus tard, le livre de Jean-Marie Mayeur (1966), La Séparation de l'Église et de l'État (titre corrigé en "des Églises », en 1991) présente une nouvelle approche. Il s'agit, pourtant, dans l'esprit de la collection où il est publié, d'un recueil de textes commentés. Mais, en donnant des extraits des débats parlementaires, en insistant sur l'importance stratégique de l'article $4^{16}$, l'auteur montre le « libéralisme croissant » à l'œuvre dans l'élaboration de la loi et ses suites. Briand redevient alors l'acteur principal de la loi de 1905 et de sa mise en œuvre apaisée, malgré l'interdiction faite aux catholiques de s'y conformer. S'il existe de l'irréversible dans la connaissance historique, on est bien là face à un tournant majeur. A posteriori, il devient étrange (sauf à analyser le poids des représentations collectives) de constater qu'il a fallu soixante ans pour retrouver ce qui était déjà perçu par un historien de l'époque!

Mayeur prouve, entre autres, "que la séparation entrait bien en application " immédiate, en ce qui concerne les libertés nouvelles de l'Église catholique, avec la nomination par le pape, en février 1906, de quatorze nouveaux évêques: "Pour

13. Debidour, 1909. Cet historien anticlérical rend compte du processus factuel de la Séparation (p. 413-498). Le rôle d'A. Briand est mis en lumière (plus de 40 références). Mais cet ouvrage est fort peu cité.

14. Avec des jugements de valeurs "cléricaux", l'ouvrage du chanoine-historien L. Capéran est bien documenté (Capéran, 1935). Dans l'autre "camp», c'est l'évitement, y compris chez Nicolet (1982), où l'histoire de la Séparation est jugée «trop connue » pour être abordée (p. 499), sans aucun renvoi bibliographique. Le recueil de textes de Nicolet, Gauthier, 1987 n'inclut pas Briand dans les 20 personnages emblématiques de l'histoire de la laïcité.

15. Pourtant A. Dansette avait inauguré une approche plus équilibrée (Dansette, 1951: 333-372) et L.V. Méjan publié une thèse consacrée à son père (Méjan, 1959).

16. Déjà mis en lumière par Debidour, que Mayeur semble ignorer (il est absent de sa bibliographie). 
la première fois dans l'histoire de l'Église de France, le Saint-Siège agissait en la matière sans se concerter avec le pouvoir civil.» (Mayeur, 1966: 122). Son étude reste, malgré tout, dans la perspective d'une histoire du catholicisme. Il estime que "l'intransigeance de Pie X était en définitive féconde; elle avait contraint le gouvernement à un libéralisme qui était bien dans l'esprit de la loi, mais dont il n'est pas sûr qu'il aurait présidé à son application sans la fermeté romaine» (ibid.: 185) ${ }^{17}$.

Outre qu'un autre historien du catholicisme, le Britannique Maurice Larkin, développera un point de vue opposé (il écrit notamment: "With he sombre encyclical, Gravissimo officii [...] Rome seemed to be inviting the very dangers it claimed to be avoiding "1 ${ }^{18}$, l'affirmation de Mayeur apparaît assez paradoxale: elle revient à mettre au crédit de Pie $\mathrm{X}$ la difficile politique libérale de Briand menée face à "l'intransigeance» papale. Si les faits sont rapportés, certaines formules tentent d'atténuer l'attitude du souverain pontife. Un exemple: quand Briand, malgré l'absence d'associations cultuelles catholiques, fait accepter par le Conseil des ministres la possibilité d'exercer «légalement» ce culte par application de la loi de 1881 sur la liberté de réunion, Mayeur écrit: «Pie X interdit d'entrer dans le jeu du gouvernement» (Mayeur, 1966: 173).

Malgré cette réserve, le rôle de Mayeur dans le renouveau historiographique de l'histoire religieuse, et de la place donnée à l'étude de la laïcité dans cette histoire, est important. Le groupe de "Créteil », qu'il constitue, donne lieu à deux ouvrages collectifs: le premier porte sur les «problèmes et méthodes » de l'histoire religieuse (Mayeur, 1975), dont j'écris, avec Claude Langlois, la conclusion (Baubérot, Langlois, 1975: 185-230); le second concerne la «libre pensée» et la «religion laïque ${ }^{19}$, thèmes alors neufs dans la production historiographique. En 1997, il publiera un autre ouvrage reprenant diverses études sur l'histoire de la laïcité en France (Mayeur, 1997).

\section{À partir de "l'observatoire protestant", l'émergence de la laïcité comme objet d'études de sociologie historique}

Je l'ai indiqué: le bilan de la sociologie de la religion en France, publié par HervieuLéger et Champion, en 1986, est centré sur les notions de "sécularisation » et de "modernité ». Cependant, lors d'un chapitre consacré au protestantisme français, les auteures écrivent: "Pour rendre compte de la logique sociale des rapports entre le protestantisme et le processus de laïcisation depuis un siècle et demi, J. Baubérot propose de distinguer deux "seuils" dans ce processus lui-même». Les trois pages suivantes sont consacrées à ces "seuils", "à la dynamique de la laïcisation" et aux «stratégies» protestantes pour y faire face (Hervieu-Léger, 1986: 279-282). Ainsi, c'est par le biais de mes travaux de sociologie historique du protestantisme que la notion de «laïcisation» fait une entrée discrète dans une synthèse écrite par deux membres du GSR.

17. Il gardera ce point de vue. Voir Mayeur, 2005: 195.

18. Larkin, 1974: 170, 173 sq. Ce livre sera publié en français en 2004 (Larkin, 2004), préfacé par Mayeur, qui écrit qu'il constitue «toujours le seul ouvrage de cette ampleur sur ce sujet» (p. 13). 19. Mayeur, 1980. Ma contribution porte sur «Le christianisme social protestant et la Libre pensée (1898-1914) », p. 231-246. 


\section{Du protestantisme à la läicité}

Quel parcours a abouti à ce résultat? Effectuons un flash-back. Mon intérêt pour la laïcité vient de loin. Lycéen, j'ai obtenu le premier prix au Concours général d'histoire en 1959. Le sujet était: «L'Église catholique en France et l'État de 1799 à 1815 » et, selon le président du jury, j'ai «fait la différence» dans la dernière partie de ma copie, en expliquant "pourquoi le Concordat ne pouvait être la solution définitive, et pourquoi il a fallu en arriver à la laïcité » ${ }^{20}$. Pour autant, c'est dans le cadre de la chaire d' " Histoire du protestantisme » de Daniel Robert ${ }^{21}$ à l'EPHE, que commencent mes activités de chercheur. Cependant, le problème de la "liberté religieuse" au XIX ${ }^{e}$ siècle constitue un des axes majeurs de ma thèse de doctorat de troisième cycle, soutenue en $1966^{22}$. Je montre que c'est la loi du 30 juin 1881 sur la liberté de réunion qui, en supprimant l'autorisation préalable, permet aux protestants de disposer pleinement de la liberté, en dehors des lieux de culte reconnus par le pouvoir. Cette liberté s'établit dans le contexte des lois de laïcité et de liberté publique, souvent conçues avant tout comme des «lois anticléricales » ${ }^{23}$. C'est, bien sûr, un point de vue (au sens littéral du terme) différent d'une approche d'histoire du catholicisme, même si l'Église catholique va également bénéficier, à partir de 1907, de la loi de 1881.

Devenu «collaborateur technique (1967) puis «assistant de recherche » (1971) de Robert à l'EPHE, je travaille sur l'histoire du mouvement protestant du Christianisme social ${ }^{24}$. J'entreprends, par ailleurs, une thèse de doctorat d'État, sous la direction de Mayeur, qui porte sur «le protestantisme face à la laïcisation de la société française $\left(\mathrm{XIX}^{\mathrm{e}}-\mathrm{XX}^{\mathrm{e}}\right.$ siècle) ». Les protestants ont été, au cours du XIX siècle, des bénéficiaires de ce processus, car l'adage "un roi, une foi, une loi » avait abouti à leur rejet. Ils en ont été également, dans la diversité de leurs tendances théologiques, des acteurs. Leur présence dans les cérémonies officielles, la reconstruction des temples (détruits sous Louis XIV), les œuvres qu'ils créent, leur défense d'un «individualisme religieux » et leur visibilité dans l'espace public déshabituent la France à se considérer comme un pays entièrement catholique. Certains d'entre eux prônent un anticléricalisme religieux, jouent un rôle important lors de la laïcisation de l'école publique et de l'instauration de la Séparation (Baubérot, 1985). Mayeur indique d'ailleurs que, dans l'orientation des « esprits vers la recherche d'une séparation véritablement libérale, les personnalités dirigeantes du protestantisme français jouèrent [...] un rôle considérable» (Mayeur, 1966: 36).

Admirant l'érudition rigoureuse des historiens du contemporain, je regrette leur faible intérêt pour la théorisation. Ainsi, au-delà de l'étude de dossiers empiriques comme l'instauration de l'école laïque, la lutte contre les congrégations

20. Baubérot, 2014a: 67. Le futur président de l'Observatoire de la laïcité, J.-L. Bianco, obtint le $1^{\text {er }}$ accessit à ce même concours.

21. Auteur, notamment, d'un ouvrage sur Les Églises réformées en France (1800-1830) (Robert, 1961).

22. "L'évangélisation protestante non concordataire et les problèmes de liberté religieuse au XIX siècle: la Société évangélique de 1833 à 1883 ", Université de Paris IV, 1966 (non publiée).

23. Ainsi la loi de 1884 rétablissant le droit au divorce peut être qualifiée de «loi anticléricale» ou de «loi de liberté publique ", suivant le point de vue auquel on se place.

24. Ce qui donne lieu à la publication d'articles et d'un ouvrage (Baubérot, 1978). 
ou la Séparation, il manque une approche globale sur le processus de laïcisation en France depuis la Révolution. Il me semble nécessaire de construire un cadre conceptuel $^{25}$. Pour mieux me former aux théorisations sociologiques, je participe aux séminaires de figures du GSR (Poulat, mais aussi Jean Séguy, Henri Desroche et François-André Isambert). Sans pouvoir le formuler de façon aussi claire, je ressens une insatisfaction identique à ce dernier, sur le peu de place accordée au politique dans la sociologie de la religion d'alors. Selon Philippe Portier, cette attitude critique est due à «l'inspiration gramscienne de [mes] années de formation " politique (Portier, 2016b: 453), les sixties, où j'ai milité à l'Union des Étudiants Communistes (UEC) puis, à partir de sa reprise en main par le PCF, dans divers mouvements d'extrême gauche.

Quoi qu'il en soit, c'est dans le cadre de la préparation de ma thèse d'État que j'entreprends de construire, à ma manière, des types idéaux diachroniques, permettant une théorisation souple: ce fut l'élaboration de la notion de deux "seuils de laïcisation » ${ }^{26}$. J'en donne les premières formulations lors de colloques tenus en 1978 (Baubérot, 1979) - année où je deviens le titulaire de la chaire Histoire et sociologie des protestantismes à l'EPHE - et 1983, dans le cadre de la SISR (Baubérot, 1983), puis dans ma thèse d'État elle-même, soutenue l'année suivante ${ }^{27}$. Le projet de loi Savary, mettant fin au dualisme scolaire au profit d'un «service public et laïque de l'éducation nationale» (SPULEN), vient d'échouer et le président du jury, l'historien Louis Girard, m'incite à me recentrer sur la seule étude du protestantisme car, affirme-t-il, «la laïcité n'intéresse plus personne» (Baubérot, 2014a: 161).

\section{La Jeune Équipe de l'EPHE «Histoire et Sociologie de la Läicité » (1988-1994)}

J'effectue pourtant le choix inverse, encouragé par une prise de contact avec la Ligue de l'enseignement. Pour cette association laïque historique ${ }^{28}$, l'échec de la loi Savary doit aboutir au renouveau de la réflexion sur la laïcité, la dégageant de sa représentation sociale dominante et réductrice: le refus de subventions publiques aux établissements privés. C'est le début d'une longue collaboration (Marineau, 2016). Ainsi, à partir de 1988, je codirige un groupe de travail de la Ligue sur "Phénomènes religieux et laïcité " ${ }^{29}$. La même année, je fonde à l'EPHE la Jeune Équipe «Histoire et sociologie de la laïcité » (HSL) qui va être reconnue par le ministère comme Équipe de recherche et d'encadrement doctoral. L'Équipe critique le paradigme de la sécularisation et veut entreprendre l'élaboration d'une conception analytique de la laïcité, distincte de son usage social, ne serait-ce que pour pouvoir étudier sociologiquement ce dernier.

25. Ma première étude sur ce sujet (Baubérot, 1972) restait très factuelle.

26. Cf. infra.

27. Le protestantisme face à la laïcisation de la société française $\mathrm{XIX}^{e}$-XX $\mathrm{X}^{e}$ siècle, thèse sur travaux pour le doctorat d'État en lettres et sciences humaines, 3 tomes, Université de Paris-Sorbonne, 1984. Extraits publiés dans Baubérot, 1985, notamment la typologisation en seuils de laïcisation, et Baubérot, 1988, notamment l'explicitation de la distinction entre sécularisation et laïcisation. 28. Qu'étudie un membre de l'Équipe HSL, J.-P. Martin. Il soutient sa thèse (sous la direction de Mayeur) en 1992 (La Ligue de l'enseignement et la République des origines à 1914) et publiera La Ligue de l'enseignement. Une histoire politique (1866-2016).

29. Deux rapports sont publiés, un sur la France (1989), un sur l'Europe (1992). 
Les premiers travaux de l'Équipe mettent en avant le concept de «pluralisme» en le dégageant du paradigme où il est analysé dans le cadre de stratégies œecuméniques qui permettent aux organisations religieuses d'autolimiter les effets déstabilisateurs de la libre concurrence du "marché religieux ». Là, c'est moins la régulation socioreligieuse interne du pluralisme qui importe que sa régulation sociopolitique externe, dans le cadre de la "construction d'une société pluraliste et laïque». Souveraineté étatique dégagée d'une conception religieuse et pluralisme des convictions ont partie liée. Dans une telle approche, les «minorités religieuses» et les "hétérodoxies" constituent un analyseur privilégié ${ }^{30}$. Ainsi en France, les minorités juive et protestante se sont situées à la jonction de la «guerre des deux France».

Je continue à travailler la notion de seuils (Baubérot, 1990a, 1990b) afin qu'elle ne serve plus seulement à caractériser les rapports du protestantisme à la laïcisation mais puisse «donner une analyse large, et modélisante, de la relation du politique et du religieux en France depuis 1789» (Portier, 2016b: 446). Avec l'Équipe, nous cherchons à expliciter la distinction entre des processus socioculturels de "sécularisation", principalement dus à des dynamiques sociales ${ }^{31}$ et des processus sociopolitiques de «laïcisation » où, idéal-typiquement, citoyenneté et religion sont dissociées et où l'identité nationale n'a pas de composante confessionnelle. Ces processus se conjuguent souvent, sans suivre forcément la même évolution. Les différencier permet d'affiner l'analyse, d'opérer des comparaisons. Des sociétés laïques peuvent présenter un degré plus faible de sécularisation que des sociétés qui maintiennent une religion nationale (Baubérot, 1994a). Logiquement, cela conduit à élargir le champ d'études à l'Europe, grâce à des travaux personnels (Champion, 1993; Baubérot, 1993), à un ouvrage collectif (Baubérot, 1994b) et à un colloque, organisé par l'Équipe HSL et l'Institut d'Études des Religions et de la Laïcité de l'Université de Bruxelles où apparait, dans le titre, le pluriel «laïcités» 32 .

Un an et demi après la création de l'Équipe, le refus par trois collégiennes de Creil d'ôter le foulard qui couvre leurs cheveux devient une «affaire» nationale. Elle fait découvrir au gouvernement Rocard qu'il n'existe pas de chaire consacrée à la laïcité dans l'enseignement supérieur français. Il s'en suit la création d'une telle chaire à l'EPHE. Renonçant à ma chaire d'Histoire et sociologie des protestantismes, je suis élu par les instances académiques à ce nouveau poste (1991) ${ }^{33}$. Peu de temps après, Danièle Hervieu-Léger, directrice des Archives, estime indispensable de construire un nouveau projet et quitte le GSR. Elle est suivie par une bonne moitié de ses membres et crée, en 1993, le Centre d'Études Interdisciplinaire des

30. Baubérot, 1991a. Il s'agit des actes d'un colloque organisé en 1989 par la Jeune Équipe HSL, avec le concours de plusieurs formations du CNRS, dont le GSR.

31. Notion plus circonscrite que dans l'optique du paradigme, mais qui gardera une certaine validité conceptuelle même quand les critiques de ce paradigme se multiplieront: Baubérot, 2003. 32. Dierkens, 1994. Nous sommes, alors, persuadés d'innover. En fait, des juristes avaient déjà, lors d'un colloque à Nice en 1959, abordé les questions de laïcité dans différents pays : Audibert, 1960. Mais nous n'avons lu cet ouvrage que tardivement.

33. Tout en continuant, occasionnellement, d'étudier le protestantisme: voir Baubérot, Zuber, 2000; Baubérot, Carbonnier-Burkard, 2016. 
Faits Religieux ou CEIFR (EHESS-CNRS) ${ }^{34}$. Le quadriennal du GSR s'achève fin 1994. La survie du Groupe semble problématique, alors que la politique du CNRS consiste à rééquilibrer le rapport Paris-Province, au profit de cette dernière. Cependant, issu de la fusion entre les autres membres restant du GSR et de l'Équipe HSL, le Groupe de Sociologie des Religions et de la Laïcité, ou GSRL

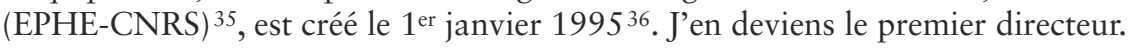

\section{Quelques pistes de sociologie de la laïcité}

Je retrace ailleurs la vie scientifique du GSRL pendant mes deux mandats de directeur ${ }^{37}$. Mais je voudrais rappeler ici la tension que nous avons dû vivre entre recherche académique et polémiques sociales. Elle est présente dès la création de la chaire en 1991: le débat autour du «voile» fait rage, et ne s'arrêtera pas. Dans ce contexte, une approche de sciences sociales souffre d'un déficit de légitimité ${ }^{38}$. Reçu par Rocard, un groupe de «philosophes républicains » lui déclare: «La laïcité, cela ne s'étudie pas, cela se vit » ${ }^{39}$. Lors de la création de la section des sciences religieuses de l'EPHE, en 1886, les autorités catholiques énonçaient une affirmation identique à propos de la religion.

Malgré ce dictat, la laïcité est étudiée. Pourtant, quand elle "fait l'actualité », le chercheur, qui est également citoyen, devient un acteur du débat public. Loin des espérances d'Habermas, ce débat fonctionne selon une logique où l'image, passée en boucle, l'emporte sur le discours, l'émotionnel et le spectaculaire sur le rationnel, l'affirmation tranchée sur une mise en perspective, médiatiquement ennuyeuse. Pour ma part, j'ai tenté de ne pas perdre ma science, à défaut de mon «âme»! (Boniface, 2013), mais je regrette que la situation calme de 1988 n'ait pas duré et pu permettre une production scientifique précédant ce climat passionnel. Celui-ci aborde la réalité sociale comme un nez de cire que l'on tord à sa guise afin de fabriquer du réel-fiction (Simone, 2013; Baubérot, 2010). Est-ce une déformation de spécialiste que d'estimer qu'il est plus facile d'énoncer des contre-vérités sur la laïcité que sur d'autres sujets, quand un organisme officiel affirme, entre autres, que Jules Ferry était protestant et que la Séparation mexicaine, qui date

\footnotetext{
34. Le CEIFR s'est plus tard regroupé avec d'autres laboratoires de l'EHESS dans le CéSor (Centre d'études en sciences sociales du religieux).

35. Tout en gardant ce sigle, il deviendra, par la suite le Groupe Sociétés, Religions, Laïcités.

36. Dans un souci d'irénisme, le GSRL a accepté l'idée que le CEIFR et lui-même constituaient les « héritiers» du GSR. Cette assertion est à moitié exacte puisque, comme le Centre de Sociologie de l'Éthique, fondé en 1978 (cf. Isambert, Ladrière, 1978), le CEIFR s'est constitué à partir de membres du GSR, mais elle est aussi à moitié fausse car le GSR a continué d'exister après la création du CEIFR et son rapport quadriennal de 1994 présente à la fois le bilan des activités du GSR (1991-1994) et le projet du GSRL, comme successeur du GSR.

37. J. Baubérot, "La sociologie de la laïcité, dépassement de la sociologie de la religion? ", à paraître dans les Actes du colloque "Autour des 20 ans du GSRL".

38. Et, souvent, le sociologue est sommé d'utiliser la notion de laïcité de façon conforme à son usage social (supposé) dominant.

39. Témoignage de M. Rocard à l'auteur. Avant «l'affaire du foulard » de Creil, aucun de ces philosophes ne s'intéressait à la laïcité.
} 
de 1859 , a «imité » la loi de $1905^{40}$ ? Autant prétendre que de Gaulle était juif et que Mitterrand a influencé Jaurès! Cela, sans parler de faux stéréotypes, tels «l'école gratuite, laïque et obligatoire » ${ }^{41}$ ou «la République une et indivisible » ${ }^{42}$. Voici, cependant, trois directions prises par mes recherches.

\section{Les seuils de laïcisation}

Au départ, deux seuils de laïcisation sont distingués. Ces périodes-type comportent chacune, à partir d'événements structurants ${ }^{43}$, un «moment» de basculement dans une nouvelle logique sociale $(1789-1802 ; 1882-1905)$ suivi d'un "moment " où cette logique s'établit progressivement, mais aussi s'use à cause de nouvelles donnes. Ainsi, en 1985, je me demande s'il ne s'est pas "produit ces quinze dernières années un mouvement relatif d'éloignement de la logique du second seuil » (Baubérot, 1985 : 303). En 1990, j'envisage l'hypothèse qu'émerge " un troisième seuil» (Baubérot, 1990b: 171 sq.; Baubérot 2000: 123 sq.) dont je précise, ensuite, qu'il s'est élaboré de 1968 à 1989 (Baubérot, 2004: 66 sq.).

Trois indicateurs, en interaction, façonnent la logique de chaque seuil, à partir du cadre général d'un État qui n'impose plus un chemin vers le «ciel » pour mieux gouverner ce qui relève du «terrestre ${ }^{44}$.

1. Le pluralisme, défini comme la prise en compte sociopolitique de la pluralité des croyances. Il est limité lors du premier seuil (les «cultes reconnus»), ouvert lors du second, avec la Séparation, et ordonné lors du troisième.

2. La socialisation morale: si l'État dispose du monopole de la violence légitime, il gouverne aussi grâce à un type de socialisation qui rend «normal » l'ordonnancement des choses. Dans ce contexte, la religion constitue une instance de socialisation morale: l'État la promeut et la contrôle ( $1^{\text {er }}$ seuil); cette socialisation, facultative, fait partie des libertés publiques mais l'ordre social est religieusement agnostique ( $2^{\mathrm{ème}}$ seuil); la séparation du "juste » et du «bien» devient un enjeu sociétal ( $3^{\text {ème }}$ seuil).

3. Les institutions comme agents du processus de socialisation: avec le premier seuil, la religion n'est plus englobante; d'autres institutions de socialisation, comme la médecine ${ }^{45}$ ou l'école, se développent et s'autonomisent progressivement (fragmentation institutionnelle); lors du second seuil, il se produit une dissociation institutionnelle ou la religion peut fonctionner en interne comme institution mais doit prendre, dans la sphère publique, une forme

40. Haut Conseil à l'Intégration, 2007: 191-194. Onze contre-vérités en quatre pages. Cf. Baubérot, 2012: 67-81.

41. Obligatoire, l'instruction a toujours pu être dispensée dans des écoles privées confessionnelles. 42. Selon la Constitution, la «République est indivisible, laïque, démocratique et sociale. [...] Elle respecte toutes les croyances ». C'est la Constitution de 1793 qui la proclamait " une et indivisible ». 43. Cette élaboration relie structurel et événementiel, pour rendre compte de l'aspect imprévisible de l'Histoire, voir Bowen, 2016.

44. Sur les seuils, voir notamment Baubérot, 2015a : 133-150; Baubérot, Milot, 2011 : 195-252. 45. Baubérot, Liogier, 2010. Exemple qui montre que la sociologie de la laïcité ne se réduit pas aux rapports religion-politique. 
analogue à l'association. Le troisième seuil est le temps d'un relatif processus de désinstitutionnalisation généralisé lié à la communication de masse. Il se produit un renouveau de la religion comme ressource culturelle.

\section{La notion de pacte laïque}

La polémique sur cette notion montre l'interférence entre démarche académique et débat social. Une première présentation en est faite en 1987. Le "pacte laïque » consiste à «intégrer », par la loi de 1905, « les libres-penseurs et les sans-religions » dans le pacte républicain, alors que, lors du régime concordataire, ils étaient des «parents pauvres » ${ }^{46}$. Cela n'a rien de formalisé, mais le rappel de cette définition succincte est ironiquement intéressant si l'on songe à l'interprétation que certains propagent depuis un bon quart de siècle: l'utilisation de l'expression «pacte laïque » prouverait que, pour moi, la loi de 1905 est le résultat d'un accord entre l’État et les religions!

En fait, c'est la version, plus travaillée, présentée en 1990 qui est mise en cause, sans référence précise à mon argumentation. Le point de départ de ma réflexion est l'inflexion qui s'est produite entre le projet de séparation d'Émile Combes et «la séparation [libérale] de Briand et Jaurès ». Aussi bien «les athées [que] les membres des différentes religions [...] chacun a de quoi être insatisfait » par la loi de 1905. C'est ce qui lui a permis «de faire vivre ensemble d'anciens ennemis irréductibles». Je poursuivais: "Il y a eu pacte pour deux raisons ». D'abord, "il ne s'est pas produit de rupture interne dans le camp laïque», bien que ses "composantes» aient eu des vues divergentes sur la Séparation: le pacte laïque est, en premier lieu, entre laïques. Ensuite, "des parlementaires qui ont voté contre la loi ont pu néanmoins [y mettre] certaines dispositions qui la rendaient moins "mauvaise" pour leur propre camp ». Deux exemples: «Les dispositions de l'article 3 sur les inventaires ", demandées par le centre-droit, même si ses effets ont été, un temps, paradoxaux; et "surtout le cas de l'article 4 » voté "grâce à des adversaires de la loi » ${ }^{47}$. Cela «aurait pu faire éclater le camp laïque. Or [...], il n'en a rien été». Les deux raisons sont donc en interaction. Je conclus: «Il y a eu compromis, il y a eu aussi victoire du camp laïque, qui a instauré, avec la séparation, une autre logique, celle [du] deuxième seuil de laïcisation.» (Baubérot, 1990b: 81 sq., 221).

Là encore, la relecture suscite un certain humour: quand on n'a plus pu se masquer l'aspect «libéral» de la loi de 1905, on m’a suggéré de remplacer l'expression «pacte laïque » par celle de «compromis». Serait-ce donc la «victoire du camp laïque que l'on aurait voulu supprimer ? ${ }^{48}$ En fait, c'est, en partie, la fin

46. J. Baubérot, in une Table-ronde: «La laïcité dans la société multiculturelle de demain " (déc. 1987), publiée dans Autogestion l'alternative PSU, 226, 1988, et republiée in Les Débats de l'ITS, 2016-4 (cf. p. 55).

47. Sur son importance, voir infra.

48. La plupart des critiques ont été allusives, se contendant d'affirmer que la loi de 1905 était un acte de l'État souverain. Une exception: Favret-Saada, 1999. L'auteure abordait la laïcité scolaire et annonçait un autre article sur la Séparation. Il n'a jamais été publié. 
de l'ouvrage, plus prospective, qui me semble avoir suscité le refus de la notion ${ }^{49}$. J'ai continué à l'utiliser, en rappelant que «pacte laïque» réunit «deux termes d'égale importance » et en dégageant des «éléments structurants » ${ }^{50}$. La notion appuie là où cela fait mal, pourrait-on écrire, car sa première signification insiste sur la pluralité des conceptions de la laïcité en France, ce qui la désubstantialise. Je typifie quatre représentations divergentes en 1905 et sept aujourd'hui (Baubérot, 2015a).

C'est un débat important à un niveau international. Certains auteurs, comme Ahmet Kuru, considèrent la laïcité française globalement comme un "assertive secularism », opposé au «passive secularism » américain (Kuru, 2009); Elisabeth Hurd remarque qu'il existe des "points aveugles » dans la laïcité française qui ne cadrent pas avec cette perspective ${ }^{51}$. Dans la typologie des différents modèles de rapports religion-État de Winfried Brugger, la laïcité française figure dans deux modèles distincts: la variante "modérée" du modèle "de rivalité ou d'hostilité entre l'État et l'Église » et celui qualifié de «séparation en théorie, considération en pratique ", plus libéral que le modèle américain de "stricte séparation » 52 . De son côté, Murat Akan, insiste sur les divergences entre acteurs de la laïcité française, avec des arguments très proches des miens (Akan, 2017). Le débat reste ouvert.

\section{De la laïcité aux laïcités}

Nous l'avons vu, dès l'Équipe HSL, l'attention portée à l'Europe a permis un décentrage à l'égard du modèle français. Selon mes collègues, le refus d'une «laïcité exception française » serait une caractéristique majeure de mon itinéraire (Blancarte, 2016; Bowen, 2016; Portier, 2016b). Elle a notamment été un élément important d'un débat intellectuel avec Catherine Kintzler ${ }^{53}$, de loin, la meilleure théoricienne d'une philosophie «républicaine» de la laïcité. Selon elle, «Locke dessine un régime de "tolérance" [...] à l'origine du modèle anglo-saxon, [la pensée] de Condorcet [est] au principe du modèle français, tel qu'il s'est établi sous la Troisième République ${ }^{54}$.

Pour moi, la laïcité française ne s'est pas construite tel un isolat (même si elle comporte des spécificités). Son histoire ressort de la problématique des «transferts culturels » (Baubérot, 2007), mise en lumière par des historiens ${ }^{55}$, qui insistent sur la circulation transnationale des représentations et son importance dans les changements sociaux. Ainsi il a existé un "modèle mexicain» lors de la Séparation française de 1905 (Baubérot, 2013) et la formulation de l'article 4 de la loi, cet article stratégiquement si important, a été emprunté aux États-Unis (Larkin, 1974: 176, 275). D’ailleurs, pour le Conseil d'État, la loi de 1905 se situe dans la

49. Pourtant, je distingue explicitement 12 "(hypo)thèses théoriques » et 13 "propositions » (cf. p. $220 s q)$.

50. Voir le chapitre «La Séparation, un pacte laïque », in Baubérot, 2000: 71-88.

51. Hurd, 2004. Elle parle de "blindness to the limitations of secularism".

52. Brugger, 2009. J. Berlinerblau estime également que la laïcité française a davantage de «limits of separation» que le secularism américain (Berlinerblau, 2012: 49).

53. Commencé dès 1990-1991: Kintzler, 1990; Baubérot, 1991b.

54. Portier, 2016b: 448 sq. Voir notamment Kintzler, 2015.

55. M. Espagne, F.-X. Guerra, J. Le Rider, M. Werner, etc. 
filiation de Locke (Conseil d'État, 2004 : 393 ; Agier-Cabanes, 2007). Sans parler de l'influence de Kant sur la morale laïque (Baubérot, 1997)...

Avec Séverine Mathieu, j’ai comparé la situation française, où, historiquement, la laïcisation prédomine et impulse la sécularisation, à celle du Royaume-Uni où la sécularisation englobe et limite une laïcisation qui existe cependant (Baubérot, Mathieu, 2002). Grâce à Micheline Milot, j'ai appris qu'un processus de "laïcisation silencieuse » existe au Canada depuis l'Acte de Québec en 177456, avant d'enquêter sur les débats actuels concernant la laïcité dans la Belle Province (Baubérot, 2008). Des ouvrages individuels ${ }^{57}$ (Baubérot, 2014b) et collectifs ${ }^{58}$ (Baubérot, Milot, 2011) ont exploré diverses laïcités (et secularisms ${ }^{59}$ ) dans différents pays. Milot, Roberto Blancarte et moi-même avons impulsé une Déclaration universelle sur la laïcité, signée par 250 chercheurs de 30 pays. Elle affirme que «la laïcité n'est l'apanage d'aucune culture, d'aucune nation, d'aucun continent. Elle peut exister dans des conjonctures où le terme n'a pas été traditionnellement utilisé ». Elle est définie comme «l'harmonisation » de trois principes: la liberté de conscience, l'autonomie du politique à l'égard des normes religieuses, la citoyenneté sans discrimination pour raison de religion ${ }^{60}$. Depuis 2007, Philippe Portier ${ }^{61}$ a repris le flambeau de la chaire, devenue «Histoire des sociologies des laïcités ». Quant à moi, je projette d'achever mon itinéraire scientifique par une étude sur la loi de 1905 en la situant dans la perspective ouverte par Patrick Boucheron dans une Histoire mondiale de la France (Boucheron, 2016).

Jean BAUBÉRoT

Groupe Sociétés, Religions, Laïcités (EPHE) jeanbauberot@hotmail.com

56. Voir ma préface à Milot, 2002: 7-11.

57. Pour ce qui me concerne: Baubérot, 2014b; Baubérot, Milot, 2011.

58. Voir notamment Baubérot, Wieviorka, 2005; Baubérot, Milot, Portier, 2014; Cady, Hurd, 2010 (ma contribution: "The Evolution of Secularism in France Between Two Civil Religions », p. 57-68); Berlinerblau, Fainferg, 2014 (mes contributions: «French Laïcité: What Does It Stand for ? " et "Laïcité and Freedom of Conscience in Pluricultural France », p. 85-94, 103-112).

59. J'ai tenté de relier les notions de secularism et de laïcité (Baubérot, 2015b).

60. Le texte de cette Déclaration, publiée en 7 langues, se trouve notamment in Baubérot, 2006b: 247-265.

61. Voir Portier, 2016c. 


\section{Bibliographie}

Audibert A. et al. (éds.), 1960, La laïcité, Paris, Presses universitaires de France.

AKan Murat, 2017, The Politics of Secularism. Religion, Diversity and Institutional Change in France and Turkey, New-York, Columbia University Press.

BAUвÉROT Jean, 1972, «Problème du protestantisme français face à la séparation des Églises et de l'État ", Études théologiques et religieuses, 3, p. 271-312.

-, 1978, Un christianisme profane? Royaume de Dieu, Socialisme et Modernité culturelle dans le périodique "chrétien social» l'Avant-garde (1899-1911), Paris, Presses universitaires de France [rééd. Archives Karéline, 2009].

-, 1979, «Le protestantisme français face à la laïcisation de l'État et de la société », Actes du colloque Les Relations entre l'État et l'Église dans les pays d'Europe latine, Bossey, Université de Genève, p. 29-32.

-, 1983, «Autonomie, concordisme ou privatisation: action sanitaire protestante en France et structure médicale et paramédicale», Actes de la XVII ${ }^{e}$ Conférence Internationale de sociologie des religions, Religions et domaine public, Londres, Paris, SISR, p. 189-206.

-, 1985, Le retour des Huguenots. La vitalité protestante, XIX ${ }^{e}$-XXe siècle, Paris-Genève, Éditions du Cerf/Labor et Fides.

-, 1988, Le protestantisme doit-il mourir? La différence protestante dans une société pluriculturelle, Paris, Éditions du Seuil

-, 1990a, La laïcité quel héritage de 1789 à nos jours? Genève, Labor et Fides.

-, 1990b, Vers un nouveau pacte laïque, Paris, Éditions du Seuil.

- (dir.), 1991a, Pluralisme et minorités religieuses, Louvain-Paris, Peeters.

-, 1991b, «Aux fondements de la laïcité scolaire. Réponse à Catherine Kintzler », Les Temps Modernes, 534, p. 163-171.

-, 1993, «La laïcité française et l'Europe », Philosophie politique, 4, p. 89-100.

-, 1994a, "Laïcité, laïcisation, sécularisation ", in Dierkens A. (éd.), Pluralismes religieux et laïcités dans l'Union européenne, Bruxelles, Éditions de l'Université de Bruxelles, p. $9-20$

- (éd.), 1994b, Religions et laïcité dans l'Europe des douze, Paris, Syros, 1994.

-, 1997, La morale laïque contre l'ordre moral sous la Troisième République, Paris, Éditions du Seuil [réédit. Archives Karéline, 2009].

-, 2000, Histoire de la laïcité française, Paris, Presses universitaires de France [devenu Histoire de la laïcité en France, 7ème éd., 2017].

-, 2003, "Construction historique et crise actuelle de la sécularisation ", in Matsuura K., Baubérot J., Bourgey A. (éd.), Les civilisations dans le regard de l'autre, II, Paris, UNESCO, p. 101-111

-, 2004, Laïcité 1905-2005, entre passion et raison, Paris, Éditions du Seuil.

-, 2006a, "Les Archives ou l'éducation à la neutralité axiologique », Archives de sciences sociales des religions, 136, p. 39-51.

-, 2006b, L'intégrisme républicain contre la laïcité, La Tour d'Aigues, L'Aube.

-, 2007, "Transferts culturels et identité nationale dans la laïcité française ", Diogène, 218, p. 18-27.

-, 2008, Une laïcité interculturelle. Le Québec avenir de la France?, La Tour d'Aigues, L'Aube. -, 2012, "La laïcité, du politique au médiatique: le "monstre doux" » in Baubérot J., La laïcité falsifiée, Paris, La Découverte, p. 103-120.

-, 2013, «Representacion e influencia de la laicidad mexicana sobre la laicidad francesa », in Blancarte R. (éd.), Las Leyes de Reforma y el Estado laico, Mexico, Colegio de Mexico, p. 217-227.

-, 2014a, Une si vive révolte, Paris, Les Éditions de l'Atelier.

-, 2014b, Les laïcités dans le monde, Paris, Presses universitaires de France, 4 ème éd.

-, 2015a, Les 7 laïcités françaises, Paris, Éditions de la Maison des sciences de l'homme. 
-, 2015b, «Sécularisation, sécularisme, laïcité dans une perspective sociologique » in Wieviorka M., Lévi-Strauss L., Lieppe G. (éd.), Penser global, Éditions de la Maison des sciences de l'homme, p. 385-399.

-, à paraître, «La sociologie de la laïcité, dépassement de la sociologie de la religion? ".

BAUbÉROT Jean, LANGlois Claude, 1975, "Problèmes et propositions d'histoire religieuse », in Mayeur J.-M. (éd.), Histoire religieuse de la France, $\mathrm{XIX}^{e}$-XX $\mathrm{X}^{e}$ siècle. Problèmes et méthodes, Paris, Beauchesne, p. 185-230.

Baubérot Jean, Zuber Valentine, 2000, Une haine oubliée. L'antiprotestantisme avant le pacte lä̈ue, Paris, Albin Michel.

Baubérot Jean, Mathieu Séverine, 2002, Religion, modernité et culture au Royaume-Uni et en France, 1800-1914, Paris, Éditions du Seuil.

Baubérot Jean, Wieviorka Michel (éd.), 2005, De la séparation des Églises et de l'État à l'avenir de la laïcité, La Tour d'Aigues, L'Aube.

BAubérot Jean, Liogier Raphaël, 2010, Sacrée médecine. Histoire et devenir d'un sanctuaire de la raison, Paris, Entrelacs.

Baubérot Jean, Milot Micheline, 2011, "Considérations critiques sur la sécularisation », in Id., Laïcités sans frontières, Paris, Éditions du Seuil, p. 159-194.

Baubérot Jean, Milot Micheline, Portier Philippe (éd.), 2014, Laïcité-laïcités. Reconfigurations et nouveaux défis (Afrique, Amériques, Europe, Japon, pays arabes), Paris, Éditions de la Maison des sciences de l'homme.

Baubérot Jean, CARbonnier-Burkard Marianne, 2016, Histoire des protestants. Une minorité en France (XVIe-XXI siècle), Paris, Ellipses.

Berger Peter, Luckmann Thomas, 1966, «Secularization and pluralism », International Yearbook for the Sociology of Religion, 2, p. 73-84.

-, "Aspects sociologiques du pluralisme», Archives de sociologie des religions, 23, 1967, p. 117-127.

Berlinerblau Jacques, 2012, How to Be Secular. A Call to Arms for Religious Freedom, Boston, Houghton Mifflin Harcourt.

Berlinerblau Jacques, Fainferg Sarah, Nou Aurora (eds.), 2014, Secularism on the Edge, New-York, Palgrave Macmillan.

Blancarte Roberto, 2016, «Baubérot et la sortie laïque de l'Hexagone », in Zuber V., Cabanel P., Liogier R. (éd.), Croire, s'engager, chercher. Autour de Jean Baubérot, du protestantisme à la laïcité, Turnhout, Brepols p. 329-356.

Boniface Pascal, 2013, "Jean Baubérot ou la laïcité caustique », in Id., Les intellectuels intègres, Paris, Jean-Claude Gawsewitch Éditeur.

Boucheron Patrick (dir.), 2016, Histoire mondiale de la France, Paris, Éditions du Seuil. Bowen John, 2016, "La laïcité décomposée» in Zuber V., Cabanel P., Liogier R. (éd.), Croire, s'engager, chercher. Autour de Jean Baubérot, du protestantisme à la laïcité, Turnhout, Brepols, p. 285-293.

BRUGGER Winfried, 2009, «From hostility through recognition to identification: statechurch models and their relationship to freedom of religion ", in Joas H., Wiegandt K. (dir.), Secularization and the World Religions, Liverpool, Liverpool University Press.

Cady Linell, Hurd Elizabeth S. (eds.), 2010, Comparative Secularisms in a Global Age, New-York, Palgrave Macmillan.

CApÉrAn Louis, 1935, L'invasion lä̈que, de l'avènement de Combes au vote de la séparation, Paris, Desclée de Brouwer.

Casanova José, 1994, Publics Religions in the Modern World, Chicago, University of Chicago Press.

ChAmpION Françoise, 1993, «Entre laïcisation et sécularisation, des rapports Églises-États dans l'Europe communautaire », Le Débat, 75-5, p. 46-72.

Cipriani Roberto, 1981 «Sécularisation ou retour du sacré», Archives de sociologie des religions, 52-2, p. 141-150. 
Conseil d’État, Un siècle de laïcité, 2004, Paris, La Documentation française.

Agier-Cabanes Isabelle, 2007, "La laïcité, exception libérale dans le modèle français », Cosmopolitiques, 16, p. 133-143

Cuchet Guillaume, Pelletier Denis, 2016, "Claude Langlois et le “moment Créteil” de l'histoire religieuse contemporaine ", in Langlois C., Le continent théologique, Rennes, Presses universitaires de Rennes.

DANSETTE Adrien, 1951, Histoire religieuse de la France contemporaine sous la Troisième République, Paris, Flammarion.

Debidour Antonin, 1909, L'Église catholique et l'État sous la III République, II, 18891906, Paris, Félix Alcan.

Dobbelaere Karel, 1981, Secularization : a multidimensionnal concept, London, Sage Publications.

-, 2009, «Laïcisation, forme manifeste de la sécularisation », in Foret F. (dir.), Politique et religion en France et en Belgique, Bruxelles, Éditions de l'Université de Bruxelles, coll. "Science politique», p. 31-46.

FAVRET-SAADA Jeanne, 1999, «La concorde fait rage: sur le nouveau pacte laïque », Les Temps modernes, 605, p. 115-160.

Galanter Marc, 1965, «Secularism East and West », Comparatives Studies in Society and History, 7-2, p. 133-159.

GugELOT Frédéric, 2016, «Intransigeantisme-libéralisme-socialisme: le triangle interprétatif de Poulat en débats", Archives de sciences sociales des religions, 176, p. 73-87.

Haut Conseil à l'Intégration, 2007, Charte de la laïcité et autres avis, Paris, La Documentation française, p. 191-194.

HeRvieu-LÉGER Danièle, 1986, Vers un nouveau christianisme? Introduction à la sociologie du christianisme occidental, avec la collaboration de F. Champion, Paris, Éditions du Cerf.

Hurd Elizabeth S., 2004, «The political authority of secularism in international relations », European Journal of International Relations, 10-2, p. 235-262.

IsAmbert François-André, 1980, «Religion et politique. Discussions internationales ", Archives de sciences sociales des religions, 49-1, p. 77-81.

IsAmbert François-André, Ladrière Paul, Terrenoire Jean-Paul, 1978, «Pour une sociologie de l'éthique", Revue française de sociologie, 19-3, p. 323-339.

KintzLER Catherine, 1990, "Aux fondements de la laïcité scolaire », Les Temps Modernes, 527, p. 82-90.

-, Penser la laïcité, Paris, Minerve, 2015.

Kunu Ahmet T., 2009, Secularism and State Policies toward Religion. The United States, France and Turkey, New York, Cambridge University Press.

LARKIN Maurice, 1974, Church and State after the Dreyfus Affair. The Separation Issue in France, London, The Macmillan Press LTD.

-, 2004, L'Église et l'État en France. 1905: la crise de la Séparation, Toulouse, Privat.

Latreille André, Rémond René, 1964, Histoire du catholicisme en France, 3, La période contemporaine, Paris, Spes.

Martin David, 1978, A General Theory of Secularization, Oxford, Basil Blackwell.

Martin Jean-Paul, 2016, La Ligue de l'enseignement. Une histoire politique (1866-2016), Rennes, Presses universitaire de Rennes.

Mayeur Jean-Marie, La Séparation de l'Église et de l'État, Paris, Julliard, 1966.

- (éd.), 1975, Histoire religieuse de la France, XIX'-XX' siècle. Problèmes et méthodes, Paris, Beauchesne.

- (éd.), 1980, Libre pensée et religion lä̈que en France de la fin du Second-Empire à la fin de la Troisième République, Strasbourg, Cerdic.

-, 1997, La question lä̈que, XIX ${ }^{e}$-XX ${ }^{e}$ siècle, Paris, Fayard.

-, 2005, La séparation des Églises et de l'État, Paris, Éditions de l'Atelier. 
MÉJAn Lucie Violette, 1959, La Séparation des Églises et de l'État. L'œuvre de Louis Méjan, dernier directeur de l'administration autonome des cultes, Paris, Presses universitaires de France.

MiLot Micheline, 2002, Laïcité dans le nouveau monde. Le cas du Québec, Turnhout, Brepols.

Morineau Michel, 2016, "Jean Baubérot et la Ligue de l'enseignement ", in Zuber V., Cabanel P., Liogier R. (éd.), Croire, s'engager, chercher. Autour de Jean Baubérot, du protestantisme à la laïcité, Turnhout, Brepols, p. 63-72.

Nicolet Claude, 1982, L'idée républicaine en France, Paris, Gallimard, 1982.

Nicolet Claude, Gauthier Guy, 1987, La laïcité en mémoire, Paris, Edilig.

Portier Philippe, 2016a, «Le "moment laïcité" dans l'œuvre d'Émile Poulat ", Archives de sciences sociales des religions, 176, p. 89-97.

-, 2016b, «Jean Baubérot et l'étude de la laïcité. Un tournant théorique », in Zuber V., Cabanel P., Liogier R. (éd.), Croire, s'engager, chercher. Autour de Jean Baubérot, du protestantisme à la laïcité, Turnhout, Brepols.

-, 2016c, L'État et les religions en France. Une sociologie historique de la laïcité, Rennes, Presses universitaires de Rennes.

Poulat Émile, 1958, «Une enquête anticléricale de pratique religieuse en Seine-et-Marne (1903) ", Archives de sociologie des religions, 6, p. 127-148.

-, 1960, «Socialisme et anticléricalisme. Une enquête socialiste internationale (1902-1903) », Archives de sociologie des religions, 10, p. 109-131.

-, 1969, «Quinze ans de vie et de travail », Archives de sociologie des religions, 28, p. 2-92.

-, 1977, Église contre bourgeoisie, Paris, Casterman.

-, 1988, Liberté, laïcité. La guerre des deux France et le principe de la modernité, Paris, Éditions du Cerf/Cujas.

-, 1994, «Catholiques sans Église», Catholica, 43.

-, 2003, Notre laïcité publique. "La France est une République laïque», Paris, Berg International.

-, 2010, Scruter la loi de 1905. La République française et la Religion, avec la collaboration de M. Gelbard, Paris, Fayard.

Robert Daniel, 1961, Les Églises réformées en France (1800-1830) Paris, Presses universitaires de France.

Simone Raffaele, 2010, Le monstre doux. L'Occident vire-t-il à droite?, Paris, Gallimard.

Sмiтн Donald Eugene, 1963, India as a Secular State, Princeton, Princeton University Press.

Tschannen Olivier, 1991, "The secularization paradigm: a systematisation ", Journal for the Scientific Study of Religion, 30-4, p. 395-415.

Weber Max, 2003 [1904-1905], L'éthique protestante et l'esprit du capitalisme suivi d'autres essais, trad. de l'allemand et présenté par Grossein J.-P., Paris, Gallimard. 